\title{
The expression of $\beta$-catenin in non-small-cell lung cancer: a clinicopathological study
}

\author{
Jeroen M A M Retera, Mathie P G Leers, Maarten A Sulzer, Paul H M H Theunissen
}

\begin{abstract}
Aims-To investigate the expression of $\beta$-catenin in non-small-cell lung cancer (NSCLC) and its clinical significance. Methods-101 patients were surgically treated for NSCLC by lobectomy or pneumectomy with systematic lymph node dissection. Follow up was available in all patients, ranging from 24 to 110 months. Immunostaining of tissue sections from primary tumours and (when present) their lymph node metastases was performed and evaluated using a monoclonal antibody against $\beta$-catenin. Correlations were investigated between $\beta$-catenin immunostaining in primary tumours and $E$-cadherin immunostaining (data available from a previous study), lymph node stage, and survival.
\end{abstract}

Results-There were significant correlations between scores for $\beta$-catenin immunostaining and $E$-cadherin immunostaining in primary tumours $(p=0.007)$, and between the $\beta$-catenin immunostaining score in primary tumours and in their lymph node metastases $(p=0.006)$. An inverse correlation was found between the $\beta$-catenin immunostaining score in primary tumours and lymph node stage N0, $\mathrm{N} 1$, or $\mathrm{N} 2(\mathrm{p}=\mathbf{0 . 0 3})$. According to the Kaplan-Meier survival estimate, the level of $\beta$-catenin expression in primary tumours was a statistically significant prognostic factor $(p=0.01)$.

Conclusions-Reduced $\beta$-catenin expression in surgically treated NSCLC is clearly associated with lymph node metastasis and an unfavourable prognosis. The existence of a functional relation between $E$-cadherin and $\beta$-catenin is supported by the results of this clinicopathological study.

(F Clin Pathol 1998;51:891-894)

Department of

Pulmonary Medicine, Atrium Medical

Centre, PO Box 4446, 6401 CX Heerlen, The Netherlands

J M A M Retera

M A Sulzer

Department of Pathology, Atrium Medical Centre M P G Leers

P H M H Theunissen

Correspondence to:

Dr P H M H Theunissen.

Accepted for publication 11 August 1998 dimeric zipper-like adhesive structures at the cell membrane to make contact with another cell. E (epithelial)-cadherin is linked to the actin cytoskeleton through cytoplasmic proteins termed $\alpha-, \beta-$ and $\gamma$-catenin. The cytoplasmic domain of E-cadherin cannot directly associate with $\alpha$-catenin but interacts with $\beta$-catenin; $\alpha$-catenin in its turn mediates the interaction with the actin filament network. ${ }^{1}$

Experimental evidence suggests that disturbance of the E-cadherin/catenin complex is crucial in the process of loss of differentiation and the onset of invasion. ${ }^{2}$ Mutations in the E-cadherin gene, localised on chromosome $16 q 22.1$, have been found in a minority of human cancers. We were unable to detect mutations of this gene in any non-small-cell lung cancers (NSCLC) (unpublished observations). The E-cadherin/catenin complex has been shown to be regulated multifactorially at multiple, mostly non-genomic, levels. Experimental data support the view that tyrosine phosphorylation of $\beta$-catenin downregulates the function of the complex which may permit invasive growth. In fact, $\beta$-catenin seems to be one of the key molecules in modulating the balance between processes of growth factor activation and signal transduction from adhesion receptors. ${ }^{3}$

In a previous study on a group of patients with NSCLC we were able to demonstrate that reduced E-cadherin expression is clearly associated with lymph node metastasis and an unfavourable prognosis. ${ }^{4}$ Our aim in this present clinicopathological study was to investigate-in almost the same group of patients as in the previous study - the expression of $\beta$-catenin in NSCLC and its clinical significance.

\section{Methods}

PATIENTS AND TUMOURS

We studied retrospectively the primary tumours of 101 patients who underwent complete resection of NSCLC (58 squamous cell carcinomas, 29 adenocarcinomas, and 14 large cell anaplastic carcinomas) between January 1989 and July 1996 in the Atrium Medical Centre Heerlen, the Netherlands. There were 86 male and 15 female patients, mean age 64 years. Mediastinoscopy was performed in all patients and did not reveal any lymph node metastases. During pulmonary resection a systematic mediastinal lymph node dissection was performed in all patients, contributing to a highly accurate pathological staging with respect to lymph node involvement. Clinicopathological stage was assigned using the TNM classification of the Union Internationale Contre le Cancer (UICC), and the 
histological typing and grading of tumours was evaluated on the basis of the World Health Organisation (WHO) criteria. To obtain comparable patient groups, only $\mathrm{T} 1$ and $\mathrm{T} 2$ tumours (tumours without extrapulmonary extension) were selected for the study. Since the majority of patients with NSCLC do not have lymph node metastases, we stopped evaluating patients with N0 stage after 1995 in order to have a balanced number of patients in each lymph node stage subgroup. Lymph node metastases were detected in 54 of 101 patients. None of the patients received neoadjuvant treatment and only patients with $\mathrm{N} 2$ disease (ipsilateral mediastinal lymph node metastasis) received radiotherapy postoperatively. Follow up data on all patients was available, ranging from 24 to 110 months.

IMMUNOHISTOCHEMISTRY

A representative tissue sample from each tumour was fixed in neutral buffered formalin $(4 \%)$ and routinely processed for paraffin embedding. From all paraffin blocks $4 \mu \mathrm{m}$ thick sections were cut and mounted on APES coated slides. For immunostaining the sections were deparaffinised in xylene and rehydrated in a descending ethanol series. Endogenous peroxidase activity was blocked by immersing the slides for 10 minutes in 3\% hydrogen peroxidase in methanol, after which they were rinsed in phosphate buffered saline (PBS; pH 7.2-7.4). Antigen retrieval was performed by placing the slides in a $0.1 \mathrm{M}$ citrate buffer $(\mathrm{pH}$ 6.0 ) and boiling for 10 minutes in a microwave oven at $750 \mathrm{~W}$. After a short cooling down period, sections were preincubated with $1 \%$ bovine serum albumin (Sigma)/PBS for 10 minutes. Tissue sections were incubated overnight at room temperature in a humidified chamber with the primary antibody $\beta$-catenin (clone $14, \mathrm{IgG}_{1}, 20 \mu \mathrm{g} / \mathrm{ml}$, Transduction Laboratories). After incubation, antibody binding was visualised by rinsing the sections in PBS

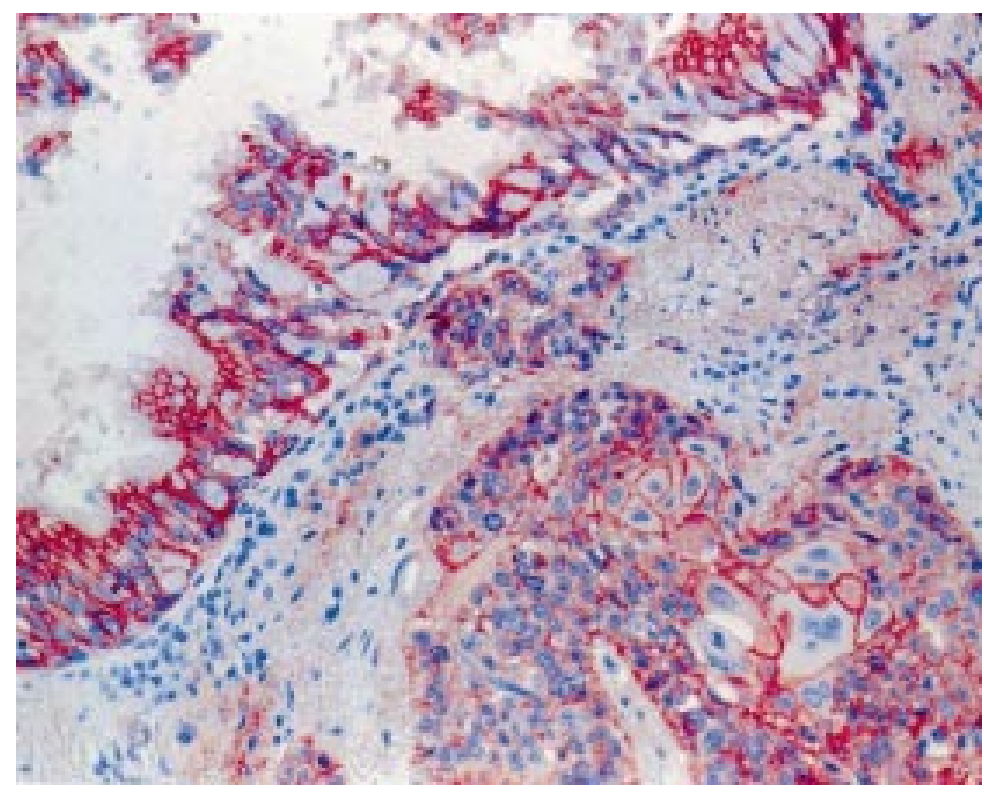

Figure 1 Example of a bronchial squamous cell carcinoma immunostained with monoclonal antibody against $\beta$-catenin. The tumour cells and the normal ciliated epithelium of the bronchus show a predominantly membranous staining pattern $(\times 183)$. and incubation with the secondary biotinylated goat antimouse immunoglobulin (1:400, Dako) for 45 minutes at room temperature. After washing in PBS, the slides were incubated with streptavidin and conjugated with horse radish peroxidase (1:600, Dako). Peroxidase activity was developed with aminoethylcarbazole (AEC) $/ 0.002 \% \quad \mathrm{H}_{2} \mathrm{O}_{2}$ solution (Sigma). Finally, the sections were counterstained with Harris's haematoxylin and embedded with Kaiser's glycerine gelatin (Merck).

Multitissue slides with epithelial and nonepithelial tissues were used as controls. In addition, normal bronchial epithelium, normal mucosal glands, and pneumocytes within the tumour section were used as positive internal controls. Cases in which this internal positive control failed to show clear staining with the $\beta$-catenin were excluded from this study (four cases). Because of intratumour heterogeneity with respect to antigen expression - as could be observed in a pilot study-results were classified semiquantitatively into three categories according to the proportion of tumour cells with immunoreactivity for $\beta$-catenin on the one hand, and the mean staining intensity of positively stained tumour cells on the other. Both cytoplasmic and membranous staining were considered positive. Cut off values were determined beforehand. Immunostaining in less than $25 \%$ of tumour cells was categorised as 1 , staining in $25-75 \%$ of tumour cells as 2 , and staining in more than $75 \%$ of the tumour cells as 3 . The mean staining intensity in every single case was estimated at low power magnification (25×) and categorized 1, 2, or 3 (weak, moderate, or strong). The total immunostaining score consisted of the product of the category for the amount of positive tumour cells $(1-3)$ and the category for staining intensity (1-3), resulting in a minimum score of 1 and a maximum of 9 . This total immunostaining score was subdivided into low scores $(\leqslant 3)$, moderate scores ( $\geqslant 4$ and $<7$ ), and high scores $(\geqslant 7)$. All slides were examined at random by two different investigators (JR and PT). In case of disagreement the slides were reviewed again and a consensus score was reached.

\section{STATISTICAL METHODS}

Correlations were analysed using Pearson's correlation coefficient and considered significant at the 0.05 level (two tailed). Life table probabilities of overall survival (that is, survival without regard to cause of death) were calculated by the Kaplan and Meier method using SPSS for Windows statistical software. Differences in survival between subgroups were compared with the log-rank test . Overall length of survival was measured starting from the day of surgery. Interobserver reproducibility was estimated using $\kappa$ statistics.

\section{Results}

We evaluated 101 primary lung tumours-58 squamous cell carcinomas, 29 adenocarcinomas, and 14 large cell anaplastic carcinomas. Lymph node metastases were absent in 47 cases and present in 54 . Of the 54 cases with lymph node metastases, 22 were staged as N1 


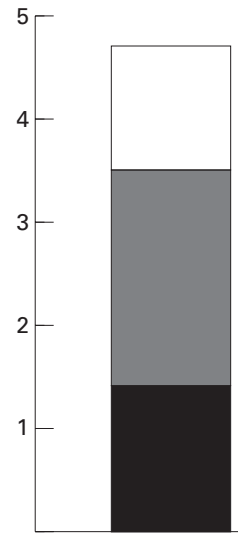

N0

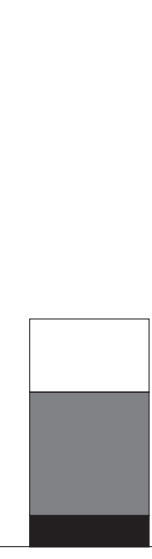

N1
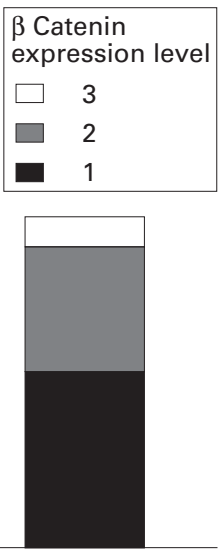

$\mathrm{N} 2$
Figure 2 Distribution of primary lung tumours according to stages N0, N1, and N2. Within each group the expression score of $\beta$-catenin in the primary tumours is shown; note that a minority of N2 tumours show a high expression level (3).

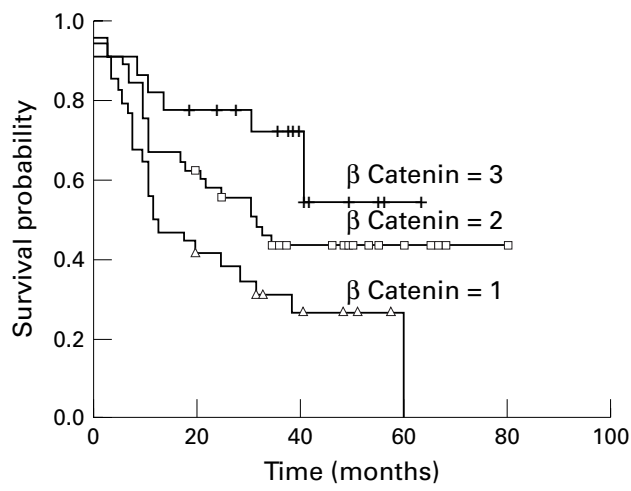

Figure 3 Kaplan-Meier survival estimate on all lung tumours $(n=101)$. Stratification on the level of $\beta$-catenin expression in the primary tumours (log-rank pooled over strata, 8.50; $p=0.01$ ).

and 32 as $\mathrm{N} 2$. In the great majority of cases, the $\beta$-catenin immunostaining of tumour cells from primary lung tumours and lymph node metastases was membranous as well as cytoplasmic (fig 1) and heterogeneously distributed in virtually all cases. In 34 of 101 primary lung tumours $(33.7 \%)$ a low immunostaining score for $\beta$-catenin was found, whereas 45 tumours $(44.6 \%)$ showed a moderate score, and 22 $(21.8 \%)$ showed a high score. There were no significant differences in $\beta$-catenin expression score between different tumour types and between tumours with different grades of differentiation.

In only 47 of the 54 lymph node metastases was enough tissue available for immunostaining. In 12 of these 47 cases $(25.5 \%)$ the immunostaining score was low, in 30 cases $(63.8 \%)$ it was moderate, and in five $(10.6 \%)$ it was high. There was a clear correlation between $\beta$-catenin immunostaining in primary tumours and staining of lymph node metastases (Pearson correlation coefficient 0.394, $\mathrm{p}=0.006)$. Interobserver agreement in scoring of the immunohistochemical staining was better in lymph node metastasis $(\kappa=0.68)$ than in primary tumours $(\kappa=0.46)$. Cases of disagreement only involved differences of one scoring level.

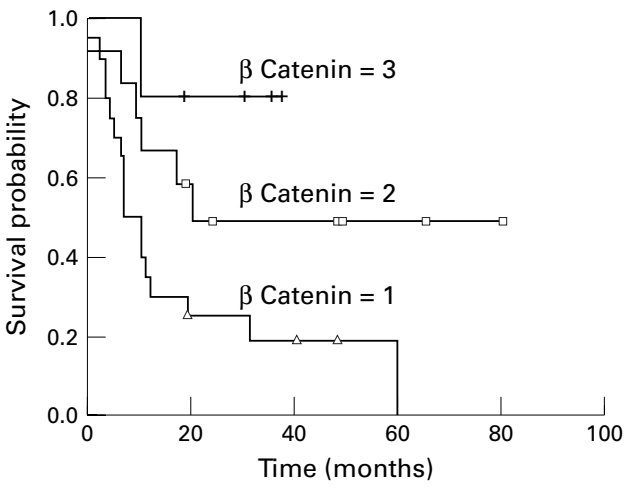

Figure 4 Kaplan-Meier survival estimate on a subset of tumours with a low expression level of $E$-cadherin $(n=37)$. Stratification on the level of $\beta$-catenin expression in the primary tumours (log-rank pooled over strata, 7.90; $p=0.02$ ).

A comparison of $\beta$-catenin immunostaining in NSCLC with E-cadherin immunostaining (data published previously ${ }^{4}$ ) showed a clear correlation between the two, as might be expected (Pearson correlation coefficient $0.268, p=0.007)$. The level of expression of $\beta$-catenin in NSCLC was inversely correlated with lymph node stage (Pearson correlation coefficient $=-0.213, \mathrm{p}=0.03)$ : high expression of $\beta$-catenin in lung tumours was found in $25.5 \%$ of cases without lymph node metastases (N0) and in only $9.4 \%$ of cases with ipsilateral mediastinal lymph node metastases (N2) (fig 2). A comparable result was found with respect to the expression level of E-cadherin in a previous study. ${ }^{4}$

In Kaplan-Meier survival estimates for patients with NSCLC, lymph node stage was a prognostic factor, as expected (log-rank pooled over strata, $6.41 ; \mathrm{p}=0.04)$. In the survival analysis, $\beta$-catenin expression level was also a significant prognostic factor (log-rank pooled over strata, 8.50; $p=0.01$; fig 3 ). In particular, the difference in survival between level 1 and level 3 was obvious (log-rank pairwise over strata, $7.32 ; \mathrm{p}=0.007$ ), as to a lesser extent was the difference between level 1 and level 2 (log-rank, $3.69 ; \mathrm{p}=0.05$ ). The mean survival time was 24.9 months for patients with lung tumours with $\beta$-catenin expression level 1, 42.8 months for level 2, and 44.1 months for level 3 . In the group of NSCLC with lymph node metastases (54 cases) preliminary results showed a trend towards better survival in cases with high $\beta$-catenin expression level in the lymph node metastasis but in the final analysis this did not reach the level of significance.

Finally, because of the interaction of two variables affecting survival in NSCLC ( $\beta$ catenin and E-cadherin), a Kaplan-Meier analysis was performed on a subset of cases with a particular level of E-cadherin expression in primary tumours and with different levels of $\beta$-catenin expression as strata. The other way round, a survival estimate was also performed on a subset of cases with a particular level of $\beta$-catenin expression in primary tumours and with different levels of E-cadherin expression as strata. The results of these Kaplan-Meier estimates showed that a significant difference in survival functions only occurred within sub- 
Table 1 Mean survival times for lung tumours with low $\beta$-catenin expression score $(n=34)$

\begin{tabular}{llll}
\hline $\begin{array}{l}\text { E-cadherin } \\
\text { expression }\end{array}$ & $n$ & $\begin{array}{l}\text { Mean survival } \\
\text { time (months) }\end{array}$ & Standard error \\
\hline Low & 20 & 18.49 & 4.93 \\
Moderate & 7 & 21.00 & 5.73 \\
High & 7 & 44.71 & 7.37 \\
\hline
\end{tabular}

Table 2 Mean survival times in lung tumours with low $E$-cadherin expression score $(n=37)$

\begin{tabular}{llll}
\hline $\begin{array}{l}\beta \text {-Catenin } \\
\text { expression }\end{array}$ & $n$ & $\begin{array}{l}\text { Mean survival } \\
\text { time (months) }\end{array}$ & Standard error \\
\hline Low & 20 & 18.49 & 4.93 \\
Moderate & 12 & 44.33 & 10.31 \\
High & 5 & 31.60 & 4.83 \\
\hline
\end{tabular}

sets of cases with low expression of $\beta$-catenin or E-cadherin. The Kaplan-Meier estimate of the subset of cases with low expression level of E-cadherin in primary lung tumours is shown in fig 4 (log-rank pooled over strata, 7.90; $\mathrm{p}=0.02)$. The results of this analysis expressed as mean survival times are summarised in tables 1 and 2 .

\section{Discussion}

From several experimental studies in the field of molecular biology, it has been shown that the function of the transmembrane cell adhesion molecule E-cadherin is dependent on $\beta$-catenin, a $92 \mathrm{kDa}$ protein which binds directly to the cytoplasmic region of E-cadherin. In a study by Kawanishi and co-workers, ${ }^{5}$ it was found that the function of E-cadherin was completely abolished in the human gastric cancer cell line HSC-39, despite of high expression of this protein, because mutations in $\beta$-catenin. An abnormal small sized $\beta$-catenin protein of $79 \mathrm{kDa}$ was immunologically identified using western blotting with a mouse monoclonal antibody against a protein fragment of $\beta$-catenin. Southern blotting of $\beta$-catenin DNA showed a mutation at genomic level.

A few years ago $\beta$-catenin was found to co-immunoprecipitate with the APC tumour suppressor protein in human colorectal tumour cell lines, whereas E-cadherin was not detectable in these complexes. ${ }^{6}$ Thus the APC-catenin complex may be affecting the transmission of contact inhibition signals or the regulation of cell adhesion.

The expression of E-cadherin and $\beta$-catenin has been studied in human carcinoma tissue samples along with normal tissue (oesophagus, stomach, and colon). ${ }^{7}$ Normal epithelium strongly expressed $\beta$-catenin, but in cancer the expression was often reduced. Co-expression of $\mathrm{E}$-cadherin and $\beta$-catenin was very common in tumours. Furthermore has been shown that reduced expression of the E-cadherin/catenin complex is associated with a poor prognosis in some tumours such as oesophageal cancer, breast cancer, and colorectal cancer. ${ }^{8-10}$
We performed the present study to determine whether if was possible to deduce a correlation between E-cadherin and $\beta$-catenin expression in lung tumours from clinical data in a group of patients who were surgically treated for NSCLC, and furthermore whether this had any clinical significance. An important aspect of the study is the fact that extensive lymph node sampling was performed during thoracotomy in this group of patients, leading to accurate lymph node staging, since this is the most important prognostic factor in NSCLC. ${ }^{11}$ From the findings in the study by Kawanishi et al mentioned above, ${ }^{5}$ we are well aware that the expression of either E-cadherin or $\beta$-catenin does not necessarily correlate with normal function of these proteins, and that because of this the results of our present study should be interpreted with caution. Accepting this limitation, our results confirm a strong correlation between E-cadherin and $\beta$-catenin expression in NSCLC. Secondly, the expression of $\beta$-catenin in primary NSCLC is inversely correlated with the presence of lymph node metastases. As lymph node metastasis is the most important prognostic factor in NSCLC, it is not very surprising that we found a significant prognostic value for $\beta$-catenin expression in these pulmonary tumours. The expression of $\beta$-catenin in lymph node metastases did not have additional prognostic value, although there was a strong correlation between the expression of this protein in primary lung tumours and in their lymph node metastases. In a previous study we found identical results for the expression of E-cadherin in NSCLC. ${ }^{4}$ The results from the present clinicopathological study support the presence of a functional relation between E-cadherin and $\beta$-catenin in NSCLC.

1 Shiozaki $\mathrm{H}$, Oka $\mathrm{H}$, Inoue $\mathrm{M}$, et al. E-cadherin mediated adhesion system in cancer cells. Cancer 1996; 77(suppl):1605-13.

2 Vermeulen S, Van Marck V, Van Hoorde L, et al. Regulation of the invasion suppressor function of the cadherin/catenin of the invasion suppressor function of the cadh

3 Jankowski JA, Bruton R, Shepherd N, et al. Cadherin and catenin biology represent a global mechanism for epithelial cancer progression. F Clin Pathol: Mol Pathol 1997;50:289 90

4 Sulzer M, Leers M, van Noord J, et al. Reduced E-cadherin expression is associated with increased lymph node metastasis and unfavorable prognosis in non-small cell cancer. Am F Resp Crit Care Med 1998;157:1319-23.

5 Kawanishi J, Kato J, Sasaki K, et al. Loss of E-cadherindependent cell-cell adhesion due to mutation of the $\beta$-catenin gene in a human cancer cell line, HSC-39. Mol Cell Biol 1995;15:1175-81.

6 Rubinfeld B, Souza B, Albert I, et al. Association of the APC

7 Takayama T, Shiozaki H, Shibamoto S, et al. Beta-catenin expression in human cancers. Am F Pathol 1996;148:39-46.

8 Krishnadath $\mathrm{K}$, Tilanus $\mathrm{H}$, van Blankenstein $\mathrm{M}$, et al. Reduced expression of the cadherin-catenin complex in esophageal adenocarcinoma co

sis. F Pathol 1997;182:331-8.
9 Hiscox S, Jiang W. Expression of E-cadherin, alpha, beta and gamma-catenin in human colorectal cancer. Anticancer Res 1997;17:1349-54

10 Zschiesche W, Schonborn I, Behrens J, et al. Expression of E-cadherin and catenins in invasive mammary carcinomas. Anticancer Res 1997;17:561-67.

11 Bollen E, van Duin P, Theunissen P, et al. Mediastinal lymph node dissection in resected lung cancer: morbidity and accuracy of staging. Ann Thorac Surg 1993;55:961-6. 